\title{
Letter to the Editor regarding "Arrhythmias in the paediatric ICU: a prospective study of the rates and predictors of arrhythmias in children without underlying cardiac disease" by Cassel-Choudhury et al.
}

Received: 12 June 2016; Accepted: 15 June 2016; First published online: 15 July 2016

Dear Dr Jacobs and Editors of Cardiology in the Young,

We read with interest the recent publication by Cassel-Choudhury et al. examining cardiac arrhythmias in the paediatric ICU. ${ }^{1}$ The characterisation of clinically important arrhythmias facilitates the care of critically ill children, and this publication represents a step towards a deeper understanding of this paediatric ICU population. We were especially attentive to the finding of an association of aminophylline with cardiac arrhythmias, including tachyarrhythmias. Although the authors specifically excluded patients with cardiac disease, we believe that our previous experience with aminophylline in the cardiac population at Stanford can complement the work published here.

Our group has particular interest and experience with the use of aminophylline in children with critical cardiac disease. We have performed a retrospective study, a randomised trial, and a pharmacokinetic (PK) study to further our understanding of aminophylline in the prevention and treatment of acute kidney injury. ${ }^{2-4}$ As an adenosine receptor antagonist, aminophylline may mitigate the vasoconstrictive acute kidney injury associated with ischaemia-reperfusion injury that commonly occurs in children after cardiac surgery for congenital cardiac disease. In our retrospective review of 31 patients who received 52 courses of aminophylline for acute kidney injury, we found an association in improved glomerular filtration and urine output and, importantly, no significant increase in cardiac arrhythmia. ${ }^{2}$ We targeted a therapeutic concentration of $5-10 \mu \mathrm{g} / \mathrm{ml}$, which is approximately half the concentration usually targeted when aminophylline is used to treat bronchoconstriction in asthmatics and significantly lower than the threshold

Correspondence to: D. M. Axelrod, MD, Department of Pediatrics, Stanford University Medical Center, 750 Welch Road, Suite 321, Palo Alto, CA 94304 United States of America. Tel: 650-723-7913; Fax 650-725-8343; E-mail: daxelrod@stanford.edu identified for increased side-effects in a previous pharmacological study. ${ }^{6}$ Even in two patients with a previous history of documented tachycardia - ventricular tachycardia and atrioventricular tachycardia - aminophylline was used safely, maintained at average concentrations of $\sim 7 \mu \mathrm{g} / \mathrm{ml}$, and did not result in exacerbation of the underlying arrhythmia.

Subsequently, we published a randomised, placebocontrolled clinical trial of aminophylline versus placebo to prevent acute kidney injury in children recovering from cardiac surgery with $\mathrm{CHD}^{3}$ In this trial, patients in the treatment arm had average therapeutic levels of $6.8 \pm 2.3 \mu \mathrm{g} / \mathrm{ml}$ and all placebo patients had undetectable levels, as expected. Our safety analysis revealed that patients receiving aminophylline did not experience more frequent side-effects. Specifically, 16 patients experienced cardiac arrhythmia overall: seven of 72 in the placebo group and nine of 72 in the treatment group ( $\mathrm{p}=\mathrm{NS}$ ). Although we did exclude patients with previous arrhythmia history, we speculate that the postoperative cardiac patients in our study with cardiopulmonary bypass exposure, intracardiac catheters, abnormal cardiac function, and intracardiac suture material were at a high baseline risk for cardiac arrhythmia. The lack of an increased rate of arrhythmia in our aminophylline-treated group suggests that aminophylline can be used safely in critically ill children recovering from cardiac surgery with cardiopulmonary bypass.

An important difference between the current publication by Cassel-Choudhury et al. and our previous study is the administration of albuterol during aminophylline therapy. Both patients who had tachyarrhythmia in the present study also received albuterol while receiving aminophylline, one of whom at a relatively high dose ( $15 \mathrm{mg}$ /hour). No pharmacological study of concomitant albuterol and aminophylline administration exists, and it may be that this combined therapy for acute asthma exposes patients to increased arrhythmia burden. 
Indeed, many confounding factors exist when evaluating the origin of complex ICU patients with illness of multiple organ systems. Table 3 from CasselChourdhury's publication illustrates this complexity, as many overlapping risk factors could contribute to the development of arrhythmia. Although the authors appropriately attempted to control for these various confounders in their regression analysis, the determination of causation remains difficult given the observational, uncontrolled study design.

In summary, we would suggest caution when concluding "that the arrhythmias were the sequelae of [aminophylline's] use". A review of experience suggests that this causative relationship is not well established. Although we are reassured that none of the events in the present report required clinical intervention or cessation of the aminophylline infusion, we also look forward to further randomised and controlled trials to confirm the safety of aminophylline use in ICU patients.

David M. Axelrod, Adam R. Frymoyer, Scott M. Sutherland Department of Pediatrics, Stanford University Medical Center, Palo Alto, California, United States of America

\section{References}

1. Cassel-Choudhury GN, Aydin SI, Toedt-Pingel I, et al. Arrhythmias in the paediatric intensive care unit: a prospective study of the rates and predictors of arrhythmias in children without underlying cardiac disease. Cardiol Young 2015; 25: 1281-1289.

2. Axelrod DM, Anglemyer AT, Sherman-Levine SF, et al. Initial experience using aminophylline to improve renal dysfunction in the pediatric cardiovascular ICU. Pediatr Crit Care Med 2014; 15: 21-27.

3. Axelrod DM, Sutherland SM, Anglemyer A, Grimm PC, Roth SJ. A double-blinded, randomized, placebo-controlled clinical trial of aminophylline to prevent acute kidney injury in children following congenital heart surgery with cardiopulmonary bypass. Pediatr Crit Care Med 2016; 17: 135-143.

4. Frymoyer A, Su F, Grimm PC, Sutherland SM, Axelrod DM. Theophylline population pharmacokinetics and dosing in children following congenital heart surgery with cardiopulmonary bypass. J Clin Pharmacol 2016; doi:10.1002/jcph.697

5. National Asthma Education and Prevention Program TEP on the $D$ and $\mathrm{M}$ of $\mathrm{A}$. Expert panel report 3: guidelines for the diagnosis and management of asthma, 2007. Retrieved January 22, 2013, from http://www-ncbi-nlm-nih-gov.laneproxy.stanford.edu/books/ NBK7232/.

6. Mulla H, Nabi F, Nichani S, Lawson G, Firmin RK, Upton DR. Population pharmacokinetics of theophylline during paediatric extracorporeal membrane oxygenation. Br J Clin Pharmacol 2003; 55: $23-31$. 